$\begin{array}{ll}\text { Research Square } & \text { Preprints are preliminary reports that have not undergone peer review. } \\ \text { They should not be considered conclusive, used to inform clinical practice, } \\ \text { or referenced by the media as validated information. }\end{array}$

\title{
Bridging the Digital Divide: A National Survey Assessing Public Readiness for Digital Health Strategies Against COVID-19 within the United Kingdom
}

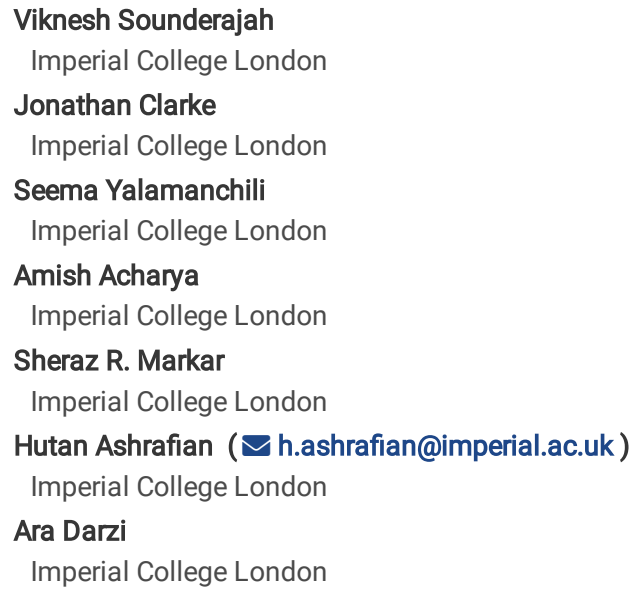




\section{Abstract}

Digital health technologies are a major feature of contemporary public health strategies, particularly in relation to the COVID-19 pandemic. However, digital initiatives risk excluding vulnerable groups, thereby propagating poor health outcomes. We assessed how groups at higher risk from COVID-19 report their relationships with key digital health initiatives in the United Kingdom. We found that those who are female, over 60 and of a lower social group are less confident in using digital information to make health decisions. Those over 40 , from lower social groups and of lower educational attainment use digital resources less often in seeking COVID-19 health information. Lastly, those over 60, from lower social groups and of lower educational attainment are less confident in distinguishing reliable digital COVID-19 information. This suggests that a 'digital first' model of COVID-19 pandemic management may exacerbate existing digital and health inequalities by reinforcing barriers to health information and public health services.

\section{Introduction}

Since emerging in December 2020, the SARS-CoV- ${ }^{1}$ virus has infected over 19.2 million people and has claimed over 715,000 lives globally ${ }^{2}$. Therapeutic options for those infected remain limited, leaving public health strategies to reduce transmission the most feasible option for effective pandemic management 3 . Accordingly, many governments have mandated unprecedented nationwide and regional behavioural measures, such as social distancing, the use of facemasks in public and extended lockdown precautions ${ }^{4}$. However, for such a public health approach to be effective, all sections of the population need to be included in communications and given equitable access to protective measures and healthcare ${ }^{5}$.

In the United Kingdom (UK), as well as a number of other countries, increased mortality from COVID-19 has been observed amongst specific cohorts, including the elderly, Black and Asian ethnic groups ${ }^{6}$, those from economically deprived areas ${ }^{7}$, and those with long-term health conditions ${ }^{8}$. The disparity in health outcomes does not appear primarily driven by SARS-CoV-2 itself, but rather that the virus amplifies pre-existing health inequalities within in society. These inequalities have been well described, stemming from the wider social determinants of health, including diet, housing, education, social networks and economic relationships ${ }^{9}$.

Governments in many countries are turning towards public-facing digital technologies to augment traditional public health strategies, particularly for the purpose of disseminating critical health information ${ }^{10}$. Whilst digital health technologies can improve the speed, reach and cost of many traditional public health measures, there are also specific barriers to their efficacy; ownership of suitable hardware, internet access and digital literacy can greatly impact effective coverage across population groups ${ }^{11}$. Crucially, these three factors, which form the concept of 'digital inclusion' ${ }^{12}$, are considered to be important social determinants of health in themselves ${ }^{13}$. Research has demonstrated that groups who are at the highest risk of digital exclusion ${ }^{14}$ are the elderly, those of Black and South Asian ethnic origin, those from deprived areas and those with low educational attainment; the same groups who suffer from the poorest COVID-19 health outcomes. It is therefore of significant concern that digital public health pathways for COVID-19, which do not account for groups at risk of digital exclusion, will further reinforce the poor health outcomes already observed within these same groups and worsen the divide in health inequality.

To date, there has been no research investigating the self-perceived preparedness for COVID-19 digital health strategies amongst the UK population. We therefore explore overall public readiness in regard to the UK digital health strategy against the COVID-19 pandemic, and, in particular, how groups at higher risk from COVID-19 report their relationships with key digital health initiatives.

\section{Methods}

\section{Survey development:}

An online survey was designed by a team of qualitative experts from YouGov, a market research company. The YouGov team used the eHealth Literacy Framework ${ }^{11}$ to devise a set of 17 core questions (Appendix 1). The eHealth Literacy Framework, the only validated framework to categorise digital health literacy (a term synonymous with eHealth literacy), consists of seven core domains; 1 ) access to digital services that work, (2) access to digital services that are suited to individual needs, (3) motivation to engage with digital services, (4) personal sense of safety and control, (5) ability to actively engage with digital services, (6) engagement in one's own health and (7) the ability to process information. Additional questions regarding proposed government and private sector contact tracing applications were devised in order to understand perception and readiness of the cohort with respect to an imminent digital technology dependent public health effort.

These 17 questions were grouped into four themes (Appendix 1); (1) confidence to independently source and use information from digital technologies to answer health related questions, (2) identify which sources of information are commonly used in gathering COVID-19 specific health information, (3) identify which information sources harbour the most trust in gathering COVID-19 specific health information and (4) quantify public opinion regarding the use of the

\section{contact tracing apps.}

Sample:

A sample of 2040 adults was achieved through YouGov's non-probabilistic sampling method. This sample is retrieved from a larger panel of more than 360,000 adults, who are registered and incentivised to participate in surveys ${ }^{15}$. The sample is representative of UK adults in terms of gender, age, ethnicity, social group, education attainment and geographical region of residence. 
Data was collected between the dates of $15^{\text {th }}$ June 2020 and $24^{\text {th }}$ June 2020 via an online survey conducted by YouGov. Participants were identified from the YouGov panel and were sent an e-mail with a survey link.

\section{Data Analysis:}

We utilised descriptive statistics to describe the sample by gender, age, ethnicity, social group, educational attainment and governmental office region respectively. Social group was categorised using the National Readership Survey (NRS) classification system and dichotomised into 'middle class' (ABC1) and 'working class' (C2DE) groups ${ }^{16}$. Education was classified as 'low' (GCSE attainment or below), 'medium' (A-level or equivalent attainment) and 'high' (university degree attainment and above). Respondent ages were grouped into young adults (18-39 years), middle-aged (40-59 year) and elderly (60+ years). Ethnicity is classed as either Caucasian or Black, Asian and minority ethnic (BAME). Government Office regions were aggregated to Southern England (London, South East and South West), Midlands (East of England, East Midlands and West Midlands), Northern England (Yorkshire and the Humber, North East and North West) and Devolved Nations (Scotland, Wales and Northern Ireland).

\section{Outcome:}

For questions with Likert-type ordinal responses, ordinal logistic regression was performed to examine the relationships between responses and the panel of demographic characteristics described above.

In order to identify discrete response types within survey domains, K-means clustering was applied to all Likert-type ordinal response variables in each domain. Data were normalised by min-max transformation and optimal clusters sizes were determined by relative maxima in silhouette and Calinski Harabasz scores and relative minima in Davies-Bouldin scores ${ }^{17-19}$. The responses of each cluster and their demographic characteristics were described. All analyses were undertaken on Stata/SE 16.0 (Stata Corporation LP, College Station, Texas, United States of America). K-means clustering was performed using Python v.3.6.8 with the scikit-learn library (version 0.23.1).

\section{Results}

A sample of 2040 adults (Table 1) was achieved through YouGov's non-probabilistic sampling method. Figure 1 is a significance map which details the directionality and the level of significance associated with responses and the panel of pre-specified demographic characteristics.

$99 \%(2024 / 2040)$ of the sample cohort have access to a personal digital device (Question 1). Smartphones and laptops/personal computers have the highest penetrance at $88 \%(1788 / 2040)$ and $84 \%(1719 / 2040)$ across the cohort respectively. $61 \%(1239 / 2040)$ of the cohort own tablet computers. Smartwatches $(211 / 2040,10 \%)$ and wearable fitness trackers $(391 / 2040,19 \%)$ were less frequently owned by respondents.

With respect to age, access to personal computers/laptops is stable through to the $70+$ age group (337/377 (89\%) in 18-29 age group compared to $215 / 259$ (83\%) in the 70+ age group). In contrast, smartphone ownership declines in the 70+ age group (359/377 (95\%) in the 18-29 age group compared to $171 / 259$ (66\%) in the $70+$ age group). Ownership of laptops/personal computers decline with lower social grade (508/571 (89\%) in AB compared to $337 / 449$ (75\%) in DE). Smartphone ownership declines with lower educational attainment groups (587/634 (93\%) in the high educational attainment group compared to $434 / 535$ (81\%) in the low educational attainment group).

$836 / 2024$ ( $41 \%$ ) of respondents state that they have used their personal digital device to access COVID-19 specific information (Question 1.1). This figure decreases with age (189/374 (51\%) between ages 18-29 compared to 50/255 (20\%) in those aged above 70$)$, social grades (274/568 (48\%) in AB compared to $145 / 442(33 \%)$ in DE) and educational attainments cohorts (329/632 (52\%) in the high educational attainment group compared to $160 / 529(30 \%)$ in the low educational attainment group). Of all personal digital device activities, instant messaging (1652/2024 (82\%)) was the most commonly utilised function, followed by accessing the news $(1476 / 2024(73 \%))$, telephone calls $(1461 / 2024(72 \%))$ and then social networking $(1447 / 2024$ (71\%)).

\section{(1) Confidence:}

$1423 / 2040$ (70\%) are confident at using online or app-based information to make personal health decisions (Question 2). In comparison to their reference counterparts, respondents who are female, over the age of 60 and of a lower social group are all significantly less confident in using online or app-based information to make personal health decisions $(p<0.01$ ) (Question 2). Those above the age of 60 are consistently significantly less confident in both sourcing and using health resources to form personal health decisions regardless of digital source (internet, apps or social media) ( $p<0.01)$ and would rather consult a clinician over the phone than an online or app-based telemedicine service $(p<0.01)$ (Question 3). Those from lower social groups and of lower educational attainment are significantly less confident at less confident at knowing where (Question 6.1) and how (Question 5.1) to use the internet to answer health questions $(p<0.01)$. There are no significant consistent findings with respect to either ethnicity or region for this domain of questions.

Four distinct clusters of responses for this domain of questions (Questions 3, 5 and 6) were identified. Panel A of Figure 2 shows the responses of each cluster to each of the constituent questions on which clustering is performed. Clusters were characterised post-hoc based on their responses as 'Digitally confident and preferring online primary care' (19\%), 'Digitally confident and preferring telephone primary care' (34\%), 'Digitally cautious and preferring online primary care' (24\%) and 'Digitally cautious and preferring telephone primary care' $(23 \%)$.

\section{(2) Sources of information:}

Respondents over the age of 40, from lower social grades and of lower educational attainment use online or app-based resources less often than their reference counterparts ( $<$ 0.01) (Question 7). 675/2040 (34\%) have not used online resources or apps to seek any COVID-19 information at all (Question 7). 
Approximately three times as many people over the age of $70(124 / 259(48 \%)$ compared to $43 / 259(16 \%))$ in the 18-39 age group would rather access health information from traditional (non-digital) media sources than relying upon digital media sources (Question 10). Those above the age of 60 are more likely to turn towards tabloid newspapers, broadsheet newspapers radio and television than their references counterparts $(p>0.01)$ whilst avoiding social media ( $p>$ 0.01). Those of lower social groups and educational attainment are less likely to use the BBC website and broadsheet newspapers (paper or online format) ( $p$ $>0.01$ ) (Questions 8 and 9). Respondents of BAME background are also more likely to engage in many digital (non-NHS websites, tabloid newspaper website, broadsheet website, social media) and traditional information sources (print tabloid and broadsheet newspapers) $(p>0.01)$ than reference counterparts (Questions 8 and 9).

Five distinct clusters of responses for this domain of questions (Question 9) were identified. Panel B of Figure 2 shows the responses of each cluster to each of the constituent questions on which clustering is performed. Clusters were characterised post-hoc based on their source of information preference; 'TV, radio and broadsheets' (12.3\%), 'TV and radio' (25.7\%), TV and tabloids' (14.8\%), 'TV only' (26.4\%) and 'No traditional media' (20.7\%).

\section{(므) Trust:}

$885 / 2040$ (43\%) cited 'trust in the information found' as the main barrier against the use of online/app-based information to guide personal health decisions, ahead of 'knowing where to find information' (406/2040 (20\%)) and 'knowing how to action the information found' (379/2040 (19\%)) (Question 4). Those above the age of $60(p>0.05)$, from lower social groups $(p>0.01)$ and of lower educational attainment $(p>0.01)$ are less confident in telling apart reliable COVID-19 information from unreliable information when encountered online or through apps (Question 12).

Amongst information sources, the NHS website has the highest trust rating (1661/2040 (81\%)) whereas social media (1325/2040 (65\%)) and tabloid newspapers $(1303 / 2040(64 \%))$ has the highest distrust rating (Question 11). However, the NHS website is not as preferred by those in lower social groups $(p>0.01)$, those of low educational attainment $(p>0.05)$, those above $60(p>0.05)$ and those of BAME backgrounds $(p>0.05)$. In addition, broadsheet newspaper sources and the BBC are not as trusted as information sources by those from low social groups and low educational attainment groups ( $p>0.01$ ).

Two distinct clusters of responses for this domain of questions (Question 11) were identified. Panel C of Figure 2 shows the responses of each cluster to each of the constituent questions on which clustering is performed. Clusters were characterised post-hoc based on their responses as either 'mistrustful of non-NHS information' (37.5\%) or 'Trusting of NHS, broadsheets and BBC' (62.5\%).

Scientific endorsement of information from figures, such as Professor Chris Whitty, is seen as the most important contributor towards trust (70\% trust rating). Despite this high rating, in comparison to their reference groups, respondents from BAME backgrounds, lower social groups, low educational attainment groups and those who reside in the Midlands are less likely to trust information that has scientific endorsement. Moreover, the government trust rating was only $40 \%$, with no one demographic either more or less inclined to trust government sourced information in comparison to the reference group. Lastly, those with a high education attainment (213/634) are twice as likely to double check information that they encounter through digital resources than those of a low education attainment (80/535) (Question 14).

\section{(4) Contact tracing:}

$832 / 2040(41 \%)$ are unlikely to engage with a digital contact tracing programme, even in the event that compliance was directly linked to easing of quarantine measures. In comparison to their respective reference groups, those above the age of 70 , of the lowest social grade and those who live in the Northern regions are significantly less likely to engage in the contact tracing programme ( $p>0.01)$ (Question 15).

With respect to industry led contact tracing apps, respondents are uncomfortable with sharing their NHS number (1524/2040 (75\%)), medical history $(1538 / 2040(75 \%))$ and location (1199/2040 (59\%)). Those aged above 60 are significantly more uncomfortable in sharing data related to age, location and medical history when using industry led apps, in comparison to their reference counterparts ( $p>0.01)$ (Question 17). In comparison, with respect to government led contact tracing apps, there is less discomfort at sharing NHS number (795/2040 (39\%)), medical history (935/2040 (46\%)) and location $(772 / 3040(38 \%))$ (Question 16). With government led contact tracing apps, those of a BAME background and lower social groups are less comfortable in sharing their location than their reference counterparts $(p>0.05)$, whereas, those over the $40+$ are more likely to share their location $(p>0.01)$.

Two distinct clusters of responses for this domain of questions (Questions 15, 16 and 17) were identified. Panel D of Figure 2 shows the responses of each cluster to each of the constituent questions on which clustering is performed. Clusters were characterised post-hoc based on their responses as either 'comfortable with apps' (59.3\%) or 'uncomfortable with apps' (40.7\%).

\section{Discussion}

This study finds that the UK has diverse preferences for where and how to access healthcare information, trust in information sources and desire to share personal information. Throughout the study, there is a consistent theme of groups at higher risk of COVID-19, specifically those above the age of 60 , those of lower socioeconomic groups and those with lower educational attainment, engaging with health information and digital resources differently to those who are younger, of higher social group and of higher educational attainment. These higher risk groups typically have greater difficulty using digital information to make informed health decisions and, perhaps by consequence, are less likely to engage with digital sources of information. Whilst the same cannot be consistently said of the BAME group, it must also be noted that the BAME umbrella encompasses a heterogenous group. This heterogeneity may explain how the views expressed did not fall consistently along economic, age or educational lines. As such viewing the attitudes and views of the BAME as a single group may not be an adequate approach ${ }^{20}$. 
The findings of this study corroborate previous reports demonstrating that almost all UK households have access to the internet, but smartphone access is much less, particularly amongst the elderly, poor and less educated ${ }^{21}$. Therefore, significant proportions of vulnerable groups are left without the requisite hardware to use a COVID-19 contact tracing app. Additionally, overall $41 \%$ report being unlikely to engage with such an app, especially if they are older or poorer. These barriers to engagement could undermine the efficacy of an app which requires at least $60 \%$ uptake ${ }^{22}$. Interestingly these groups also report reduced trust in providing personal data to the tech companies rather than the government, although it was the UK government sanctioned app with centralized data that had to be dropped in favour a joint Apple and Google option using decentralized data following privacy and security concerns ${ }^{23}$.

Amongst those with digital devices, there is a preference to use them for interpersonal communication and social media, rather than for accessing information relating to COVID-19 or related health services. This is particularly true of the elderly or lower socioeconomic groups, who prefer television or print media for COVID-19 updates. Attempts to modify user behaviour to increase access and efficacy of digital health strategies will also need to overcome a lack of confidence, particularly that expressed by women, the elderly and those in lower socioeconomic groups.

Overall, the findings suggest that a 'digital first' model of COVID-19 pandemic management has the significant potential to widen existing inequalities brought about by COVID-19 by reinforcing barriers to accessing health information and services. Both within the UK and wider settings, factors resulting in unequal access of health services have been well described ${ }^{24,25}$. These higher risk groups often include poorer communities, the less literate, the elderly and the marginalised such as homeless people, migrant workers, asylum seekers and sex-workers. Unfortunately, many of these groups are also at greater risk of COVID-19 due to the intersection with poverty and poor healthcare access ${ }^{25}$.

Prior to the COVID-19 pandemic, digital exclusion through reduced access to infrastructure and digital literacy had been viewed as key social determinant of health and national commitments had been made to target the issue ${ }^{12}$. Whilst our finding of digital inequality within the study population is not novel itself, we contribute to increasing evidence suggesting that digital inequality also potentiates vulnerability to the pandemic, thereby further increasing health inequalities ${ }^{13}$.

Crucially, the inability to promptly access and understand online information prevents individuals from taking the right protective steps against COVID-19. Despite daily briefings led by government officials, real-time updates and explanatory information are provided through carefully curated digital resources created by governments and non-governmental organisations, such as the World Health Organisation. Our study shows significant variation in confidence and trust in online healthcare information across ages, education levels and social grades. Worryingly, those struggling to trust in official sources or discern amongst digital sources are also vulnerable to the propagation of misinformation ${ }^{26}$; with notable COVID-19 specific examples relating to the $5 \mathrm{G}$ network 27 and ingestion of disinfectant ${ }^{28}$. In the short term, engaging communities with lower digital health literacy through more traditional means, such as telephone or posted material, may help bridge the gap whilst longer term strategies are devised. Longer term strategies will need to centre around improved infrastructure and educational programmes addressing digital literacy ${ }^{29}$.

Failing to consider how digital interventions amplify pre-pandemic health inequalities, inequalities driven by the pandemic and inequalities of digital exclusion could be disastrous. The British population appears divided in how confidently they acquire healthcare information, where they source this information and the extent to which they trust it. While trends exist in relation to age, socioeconomic status, educational background and ethnicity, clustering of responses reveals a lack of consensus across key issues relating to the acquisition and consumption of digital healthcare data. The presence of this variation in responses suggests that there is unlikely to be a 'one-size-fits-all' digital strategy which provides equitable coverage across all regions and populations. By extension, there is equally unlikely to be a panacea eliminating digital inequality. Strategies require a holistic approach and collaboration with stakeholders and advocates from government, education structures, health sector and the technology industry to find sustainable and inclusive solutions. Such an inclusive strategy, potentially spearheaded by national digital infrastructure organisations such as NHS Digital or NHS X, could have the potential to close, rather than widen, digital inequality for this pandemic and beyond.

\section{Limitations:}

The sampling methodology employed by YouGov is both a strength and limitation of the study. The non-probabilistic method employed allowed for the prompt and cost-effective delivery of a prespecified sample size from segments of the population, who are traditionally difficult to engage in qualitative research. However, non-probabilistic sampling precludes nonresponse bias calculations and therefore a higher degree of bias than probabilistic sampling. Additionally, this cross-sectional survey, provides a snapshot of people's preferences, rather than how sentiments evolve over time. Indeed public trust in entities such as government varies over the course of a crisis and could provide some explanation for the low government net trust rating (40\%) ${ }^{30}$. Study data did not include comorbidities of respondents and so exploration of this COVID-vulnerable group could not be performed. Furthermore, the YouGov survey is also unlikely to have accessed proportionate numbers of marginalised people such as migrant workers, the homeless and sex-workers who are at risk of COVID-19 and have poor access to healthcare and digital interventions ${ }^{12,24,25}$. Indeed, those without internet access will also not have been able to participate in the study.

\section{Conclusion}

This study demonstrates the varied preparedness for digital health strategies to contain COVID-19 in the UK. It also highlights how the 'digital first' model of health information dissemination, telemedicine portals and smartphone app-centred contact tracing, have significant potential to exacerbate existing inequalities by reinforcing barriers to accessing health information and services in vulnerable groups. This may, in turn, worsen outcomes for those at higher risk of COVID-19 as these same groups report reduced preparedness with respect to digital public health interventions.

Page 5/10 
Given the importance of maintaining low transmission rates across all regions and population groups, there is an urgent need for key decision makers to consider further investment in multifaceted strategies to mitigate for this possibility. This includes multi-modal dissemination of health information, digital health literacy programmes and revised contact tracing strategies for at-risk groups in order to maintain adequate universal coverage and bridge the digital

divide. By empowering patients, particularly those at highest risk, with such targeted schemes, public health strategies will have the greatest chance of limiting the effects of a 'second wave' and ensuring that the R number remains well contained.

\section{Declarations}

\section{Acknowledgements:}

We would like to thank Sophie Webb and Gavin Ellison of the YouGov team for their kind assistance

\section{Funding:}

JC acknowledges support from The Wellcome Trust grant 215938/Z/19/Z which supports the Sir Henry Wellcome Postdoctoral fellowship.

Infrastructure support for this research was provided by the NIHR Imperial Biomedical Research Centre (BRC).

\section{Declaration of interest:}

The corresponding author affirms that this manuscript is an honest, accurate, and transparent account of the study being reported; that no important aspects of the study have been omitted; and that any discrepancies from the study as planned have been explained.

\section{Contributorship statement:}

Viknesh Sounderajah, Amish Acharya, Sheraz Markar, Hutan Ashrafian and Ara Darzi planned the study. Viknesh Sounderajah, Jonathan Clarke, Amish Acharya and Hutan Ashrafian created the survey questions. Seema Yalamanchili and Jonathan Clarke conducted the statistical analysis. Viknesh Sounderajah, Jonathan Clarke and Seema Yalamanchili all contributed to the writing of the manuscript. Sheraz Markar, Hutan Ashrafian and Ara Darzi undertook the senior review of the work upon completion of the manuscript preparation.

\section{Other:}

This is a non-clinical population survey audit of public respondents (not patients or vulnerable individuals) that constitutes an observation of usual practice. This was therefore except from national or institutional ethics review according to the UK HRA guidelines and our University Research Office.

YouGov provided the datasets to The Institute of Global Health Innovation.

Data is publicly available upon request.

Patients and members of the public were not involved in the design, reporting or conduct of the study.

\section{References}

1. World Health Organization. Coronavirus (COVID-19) events as they happen. WHO https://www.who.int/emergencies/diseases/novel-coronavirus2019/events-as-they-happen (2020).

2. COVID-19 Map - Johns Hopkins Coronavirus Resource Center. https://coronavirus.jhu.edu/map.html.

3. Zhu, N. et al. A novel coronavirus from patients with pneumonia in China, 2019. N. Engl. J. Med. 382, 727-733 (2020).

4. Chu, D. K. et al. Physical distancing, face masks, and eye protection to prevent person-to-person transmission of SARS-CoV-2 and COVID-19: a systematic review and meta-analysis. www.thelancet.com 395, (2020).

5. Armitage, R. \& Nellums, L. B. The COVID-19 response must be disability inclusive. The Lancet Public Health vol. 5 e257 (2020).

6. Pan, D. et al. The impact of ethnicity on clinical outcomes in COVID-19: A systematic review. EClinicalMedicine 23, (2020).

7. Chaudhry, R., Dranitsaris, G., Mubashir, T., Bartoszko, J. \& Riazi, S. A country level analysis measuring the impact of government actions, country preparedness and socioeconomic factors on COVID-19 mortality and related health outcomes. EClinicalMedicine 0, 100464 (2020).

8. Clark, A. et al. Global, regional, and national estimates of the population at increased risk of severe COVID-19 due to underlying health conditions in 2020: a modelling study. Lancet Glob. Heal. 8, e1003-e1017 (2020).

9. Marmot, M. \& Allen, J. COVID-19: exposing and amplifying inequalities. doi:10.1136/jech-2020-214720.

10. Whitelaw, S., Mamas, M. A., Topol, E. \& Van Spall, H. G. C. Applications of digital technology in COVID-19 pandemic planning and response. Lancet Digit. Heal. 0, (2020).

11. Kayser, L. et al. A multidimensional tool based on the eHealth Literacy Framework: Development and initial validity testing of the eHealth Literacy Questionnaire (eHLQ). J. Med. Internet Res. 20, e36 (2018).

12. What we mean by digital inclusion - NHS Digital. https://digital.nhs.uk/about-nhs-digital/our-work/digital-inclusion/what-digital-inclusion-is.

13. Beaunoyer, E., Dupéré, S. \& Guitton, M. J. COVID-19 and digital inequalities: Reciprocal impacts and mitigation strategies. Comput. Human Behav. 111, 106424 (2020). 
14. Exploring the UK's digital divide - Office for National Statistics.

https://www.ons.gov.uk/peoplepopulationandcommunity/householdcharacteristics/homeinternetandsocialmediausage/articles/exploringtheuksdigitaldi 03-04.

15. YouGov | Panel Methodology. https://yougov.co.uk/about/panel-methodology/.

16. Social Grade | National Readership Survey. http://www.nrs.co.uk/nrs-print/lifestyle-and-classification-data/social-grade/.

17. Caliñski, T. \& Harabasz, J. A Dendrite Method Foe Cluster Analysis. Commun. Stat. 3, 1-27 (1974).

18. Rousseeuw, P. J. Silhouettes: A graphical aid to the interpretation and validation of cluster analysis. J. Comput. Appl. Math. 20, 53-65 (1987).

19. Davies, D. L. \& Bouldin, D. W. A Cluster Separation Measure. IEEE Trans. Pattern Anal. Mach. Intell. PAMI-1, 224-227 (1979).

20. The problem with 'BAME' within a UK public health context- one size really doesn't fit all - Power to Persuade. http://www.powertopersuade.org.au/blog/the-problem-with-bame-within-a-uk-public-health-context-one-size-really-doesnt-fit-allnbsp/31/5/2019.

21. Internet access - households and individuals, Great Britain Statistical bulletins - Office for National Statistics. https://www.ons.gov.uk/peoplepopulationandcommunity/householdcharacteristics/homeinternetandsocialmediausage/bulletins/internetaccesshouseho

22. Ferretti, L. et al. Quantifying SARS-CoV-2 transmission suggests epidemic control with digital contact tracing. Science (80-. ). 368, (2020).

23. The UK's contact tracing app fiasco is a master class in mismanagement I MIT Technology Review. https://www.technologyreview.com/2020/06/19/1004190/uk-covid-contact-tracing-app-fiasco/.

24. Local action on health inequalities. www.instituteofhealthequity.org (2015).

25. Inequalities in health (e.g. by region, ethnicity, soci-economic position or gender) and in access to health care, including their causes | Health Knowledge. https://www.healthknowledge.org.uk/public-health-textbook/medical-sociology-policy-economics/4c-equality-equity-policy/inequalities-distribution.

26. Ahmed, W., Vidal-Alaball, J., Downing, J. \& Seguí, F. L. COVID-19 and the 5 G conspiracy theory: Social network analysis of twitter data. J. Med. Internet Res. 22, (2020).

27. Mast fire probe amid 5 G coronavirus claims - BBC News. https://www.bbc.co.uk/news/uk-england-52164358.

28. Coronavirus: Two men in US drink disinfectants in bid to prevent Covid-19 | The Independent.

https://www.independent.co.uk/news/world/americas/coronavirus-disinfectant-drink-bleach-covid-19-atlanta-georgia-a9489566.html.

29. Wardle, C. \& Derakhshan, H. Information Disorder: Toward an interdisciplinary framework for research and policy making. Rep. to Counc. Eur. 108 (2017).

30. Fancourt, D., Steptoe, A. \& Wright, L. The Cummings effect: politics, trust, and behaviours during the COVID-19 pandemic. Lancet 0, (2020).

\section{Table}




\begin{tabular}{|c|c|c|c|}
\hline \multicolumn{2}{|l|}{ Total } & Number $(n=2040)$ & $\begin{array}{l}\text { Percentage } \\
(\%)\end{array}$ \\
\hline \multirow[t]{2}{*}{ Gender } & Male & 990 & $49 \%$ \\
\hline & Female & 1050 & $51 \%$ \\
\hline \multirow[t]{6}{*}{ Age } & $18-29$ & 377 & $18 \%$ \\
\hline & $30-39$ & 369 & $18 \%$ \\
\hline & $40-49$ & 347 & $17 \%$ \\
\hline & $50-59$ & 284 & $14 \%$ \\
\hline & $60-69$ & 356 & $17 \%$ \\
\hline & $70+$ & 259 & $13 \%$ \\
\hline \multirow[t]{4}{*}{ Social Grade } & $A B$ & 571 & $28 \%$ \\
\hline & C1 & 592 & $29 \%$ \\
\hline & $\mathrm{C} 2$ & 428 & $21 \%$ \\
\hline & $\mathrm{DE}$ & 449 & $22 \%$ \\
\hline \multirow[t]{3}{*}{ Educational attainment } & Low & 535 & $26 \%$ \\
\hline & Medium & 871 & $43 \%$ \\
\hline & High & 634 & $31 \%$ \\
\hline \multirow[t]{12}{*}{ Region } & North East & 72 & $4 \%$ \\
\hline & North West & 225 & $11 \%$ \\
\hline & Yorkshire and the Humber & 178 & $9 \%$ \\
\hline & East Midlands & 162 & $8 \%$ \\
\hline & West Midlands & 164 & $8 \%$ \\
\hline & East of England & 168 & $8 \%$ \\
\hline & London & 268 & $13 \%$ \\
\hline & South East & 285 & $14 \%$ \\
\hline & South West & 191 & $9 \%$ \\
\hline & Wales & 98 & $5 \%$ \\
\hline & Scotland & 172 & $8 \%$ \\
\hline & Northern Ireland & 57 & $3 \%$ \\
\hline \multirow[t]{2}{*}{ Ethnicity } & White & 1754 & $86 \%$ \\
\hline & BAME & 286 & $14 \%$ \\
\hline
\end{tabular}

Table 1: Survey respondent demographics table

\section{Figures}




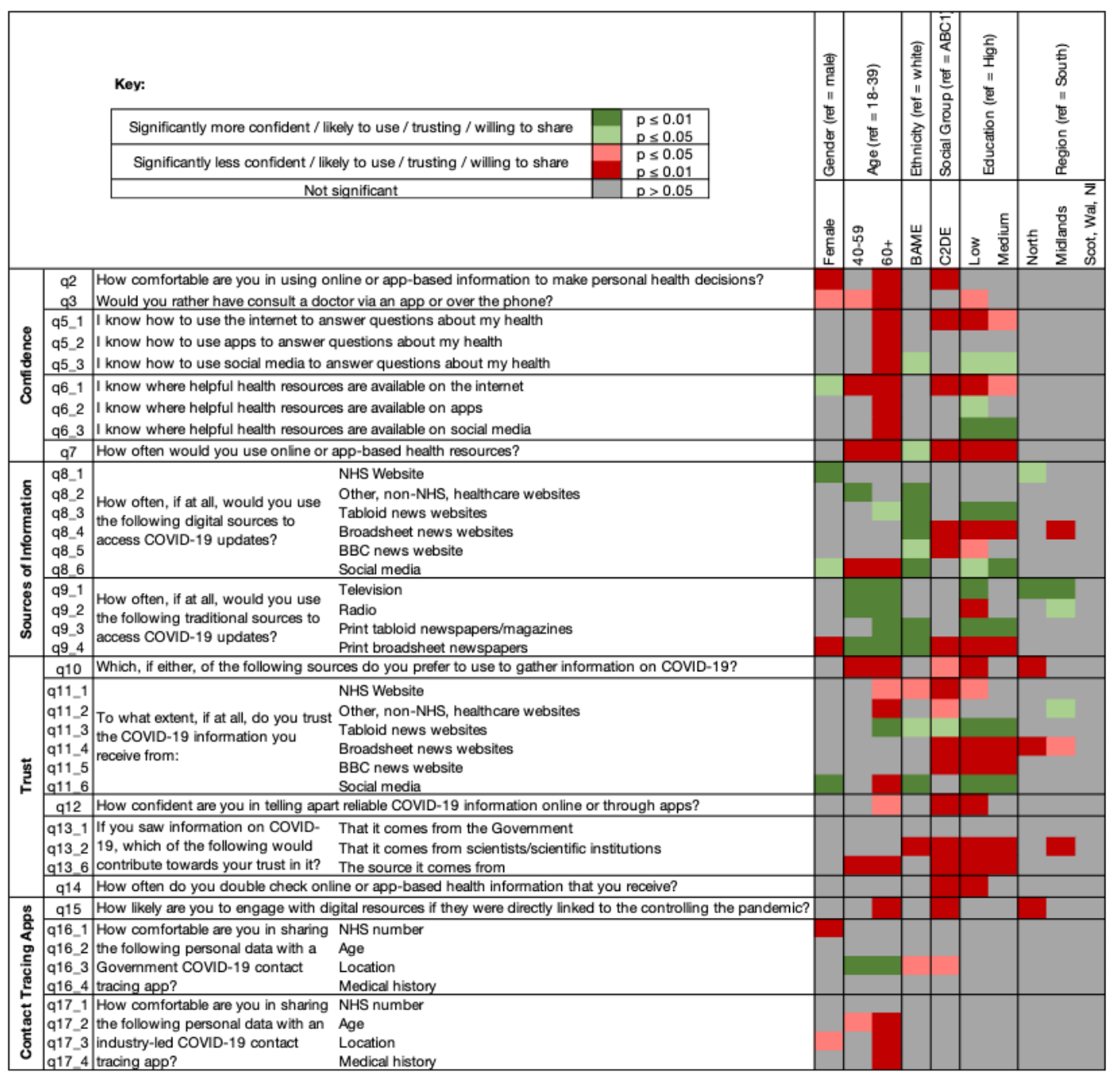

\section{Figure 1}

A significance map detailing directionality and significance of relationships between responses and the panel of demographic characteristics 

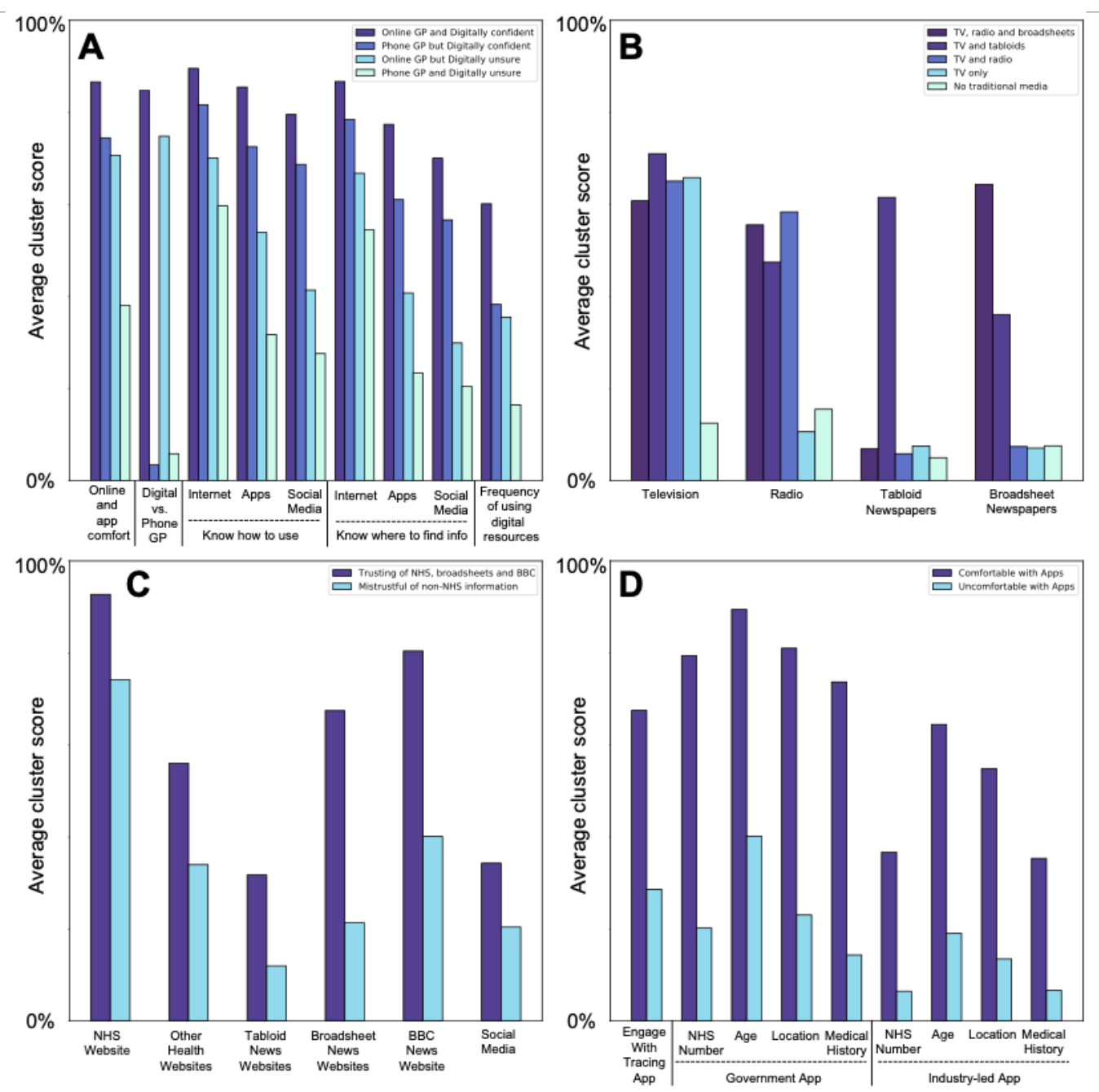

Figure 2

4 bar graphs (labelled Panel A, B, C and D) detailing discrete response types within survey domains, achieved through K-means cluster scores

\section{Supplementary Files}

This is a list of supplementary files associated with this preprint. Click to download.

- Appendix1.docx 